\title{
INFLUÊNCIA DOS PARÂMETROS DE TRATAMENTO NO RETORNO ELÁSTICO DA LIGA DE ALUMÍNIO AA7475 EM CONDIÇÕES DE CONFORMAÇÃO SOB FLUÊNCIA E ENVELHECIMENTO*
}

Leandro Wiltemburg Araújo ${ }^{1}$ Maurício de Carvalho Silva²

\section{Resumo}

Em Conformação sob Fluência com Envelhecimento, do inglês Creep Age Forming (CAF), o material previamente solubilizado e temperado é carregado elasticamente (normalmente por flexão) e subsequentemente aquecido à temperatura de tratamento térmico de envelhecimento. Basicamente, a conformação se dá por meio de deformações provocadas simultaneamente pela tensão aplicada ao longo do tempo (fluência) e endurecimento por precipitação (envelhecimento). Sendo assim, pretende-se avaliar os efeitos dos parâmetros mencionados no retorno elástico, como tempo, temperatura, retorno elástico e propriedades mecânicas. Portanto foi avaliado, a variação do retorno elástico (springback) em função do tempo para a liga de alumínio AA7475 submetida a um carregamento de flexão durante o envelhecimento realizado em duas temperaturas diferentes $\left(120^{\circ} \mathrm{C}\right.$ e $\left.150^{\circ} \mathrm{C}\right)$. Foi possível observar que quanto menor o tempo e a temperatura de envelhecimento, maior o retorno elástico. Portanto, quanto maior o retorno elástico da peça, mais próxima ela estará do seu formato original antes da conformação [1]. Em relação à dureza, foi observado que quando o material é submetido a uma temperatura de $120^{\circ} \mathrm{C}$ obtém-se um aumento significativo de dureza Vickers com o passar do tempo (atribuída a precipitação refinada) e a $150^{\circ} \mathrm{C}$ não ocorre esse aumento significativo dessa propriedade mecânica (atribuído a precipitação mais grosseira).

Palavras-chave: Conformação; Envelhecimento; Fluência; Retorno elástico.

\section{INFLUENCE OF TREATMENT PARAMETERS IN SPRINGBACK OF ALUMINUM ALLOY AA7475 IN TERMS OF CREEP AGE FORMING}

\section{Abstract}

In Creep Age Forming (CAF), the material, previously solubilized and seasoned, is loaded elastically (usually by flexion) and subsequently it is heated to temperature of heat treatment. Basically, the conformation happens to deformations caused by tension that are applied simultaneously over time (creep) and by precipitation hardening (aging). Thus, we intend to evaluate the effects of the parameters mentioned in springback, as time, temperature, springback and mechanical properties. Therefore was evaluated, the variation of springback versus time for the aluminum alloy AA7475 subjected to a load bending achieved during aging at two different temperatures $\left(120^{\circ} \mathrm{C}\right.$ and $\left.150^{\circ} \mathrm{C}\right)$. It was possible to see that the smaller time and aging temperature, the bigger the springback. Therefore, the greater the elastic return of the part, the closer it will be to its original shape before the conformation[1]. Regarding the hardness, it was observed that when the material is subjected to a temperature of $120^{\circ} \mathrm{C}$, it gains a significant increase of Vickers hardness with over time (assigned to fine precipitation) and with $150^{\circ} \mathrm{C}$, it doesn't occur a significant increase this mechanical property (assigned the grossest precipitation).

Keywords: Conformation; Aging; Creep; Springback.

Engenharia Mecânica, estudante, Departamento de Engenharia Mecânica, FEl, São Bernardo do Campo, São Paulo, Brasil.

2 Engenharia Mecânica, Doutor, Orientador, Departamento de Engenharia Mecânica e de Materiais, FEl, São Bernardo do Campo, São Paulo, Brasil. 


\section{INTRODUÇÃO}

As ligas de alumínio da série 7000 apresentam-se como materiais de grande utilização na indústria aeroespacial. $\mathrm{Na}$ indústria aeronáutica, cerca de $70 \%$ da estrutura do avião é composta de alumínio. Rotas clássicas de processamento para obtenção de estruturas de aeronaves envolvem, por exemplo, forjamento e tratamentos térmicos conduzidos em chapas grossas. Tratamentos térmicos de têmpera a partir da temperatura de solubilização conduzido em chapas de grandes dimensões gera um alto nível de tensões residuais devido ao gradiente de temperatura ao longo da espessura (deformação plástica heterogênea). Após esta etapa, a peça passa por um processo de usinagem para obtenção da forma desejada e a seguir suas tensões internas são parcialmente reduzidas por deformação plástica. Na sequência, o componente passa pelo tratamento térmico de envelhecimento para obtenção da propriedade mecânica projetada. Mesmo após todas estas operações, o nível de tensões residuais permanece alto e isto pode prejudicar, por exemplo, a vida à fadiga deste tipo de componente. Devido as constantes necessidades da indústria aeronáutica em relação à melhoria contínua de seus produtos vem surgindo inúmeros processos de fabricação dentre os quais se destaca a Conformação sob Fluência com Envelhecimento, do inglês Creep Age Forming (CAF). Trata-se de uma rota alternativa de processamento para fabricação de painéis e asas de aeronaves [2]. Este é um processo de fabricação que vem despertando interesse na indústria aeronáutica pelo potencial de obtenção de peças curvas com geometrias complexas. Neste processo, o ciclo de tratamento térmico do material é utilizado para, simultaneamente ao envelhecimento, relaxar tensões externas aplicadas, por mecanismos de fluência. Com isso, utilizando-se tempo e temperatura adequados pode-se obter o componente com propriedades mecânicas e formas desejadas.

Conformação por fluência com envelhecimento é um novo método de conformação baseado em fenômenos de fluência e relaxação que ocorrem conjuntamente com o envelhecimento artificial do metal. Foi desenvolvido para manufatura de painéis em ligas de alumínio tratáveis termicamente, particularmente painéis aeronáuticos, que requerem grande precisão e possibilidade de fabricação de peças com curvaturas complexas. Diferente de outros métodos de fabricação, como jateamento com granalha (shot-peening), estiramento, estampagem e trefilação, componentes feitos por CAF exigem menores tensões para a conformação e possuem menores tensões residuais, que por um lado diminuem a chance de ruptura e instabilidade plástica durante a fabricação e por outro aumentam sua resistência tanto à fadiga quanto à corrosão sob tensão [3].

Após ser realizado o processo CAF, as tensões de conformação tendem a relaxar devido ao descarregamento das cargas impostas. Na fase de descarga, a placa de alumínio tende a sofrer uma recuperação elástica, sabendo que os níveis de tensões se estabelecem abaixo do limite de escoamento do material, a partir da curvatura do ferramental. Esse retorno elástico, do inglês "springback", pode ser superior a 70\% da flecha inicial da chapa a ser conformada e a sua previsão é essencial para o sucesso do processo [4].

Para a aplicação correta do processo CAF é necessário conhecer, portanto, o nível de tensão (dentro do regime elástico) e também a temperatura que serão aplicados uma vez que ambos podem influenciar tanto na precipitação de partículas quanto na fluência do material, o que implica em propriedades e formas diferentes. 
Na Figura 1, mostra-se a definição do retorno elástico, o qual é quantificado por um fator $\mathrm{R}$, em que tem-se a relação de $\delta_{\text {Máx }}$ (distância formada entre a peça que foi ensaiada e a superfície da ferramenta de conformação) com $\delta o$ (distância da peça de trabalho até a ferramenta de conformação). Se $\mathrm{R}$ é igual a 1 , a peça retorna totalmente ao seu formato original, enquanto que $R$ é igual a 0 , não há retorno elástico (springback), ou seja, a peça possui o mesmo raio de curvatura que a ferramenta de conformação.

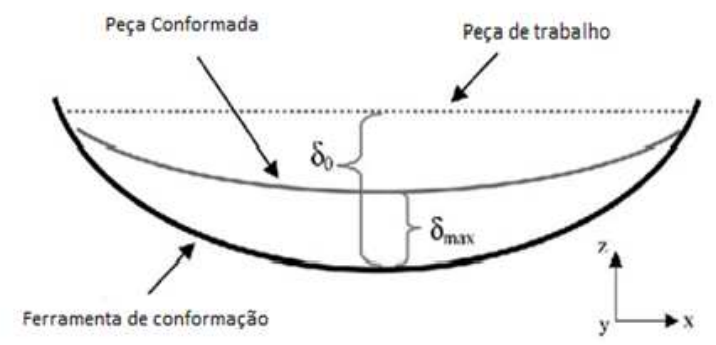

Figura 1: Definição de Springback: fator $\left(\mathbf{R}=\frac{\delta_{\text {Máx }}}{\delta_{0}}\right)$. Onde $R=0$ representa ausência de retorno elástico, $R=1$ representa totalmente elástica.

O processo CAF foi tema de estudo num primeiro projeto de iniciação científica (PBIC082/12), desenvolvido pelo aluno Marcello Vertamatti Mergulhão que finalizou seus trabalhos em outubro de 2013 [5]. Neste estudo preliminar, apesar de não ter sido o objetivo central do trabalho, notou-se que os parâmetros de tratamento térmico tais como tempo e temperatura influenciam sobremaneira na forma final da peça submetida a um carregamento de flexão em condições de conformação sob fluência e envelhecimento. Por exemplo, quanto menores tempo e temperatura maior será o retorno elástico da peça (ou seja, quanto maior for o retorno elástico da peça mais próxima esta estará do seu formato original antes da conformação).

Sendo assim, como complemento do primeiro projeto, pretende-se avaliar de forma mais detalhada os efeitos dos parâmetros mencionados no retorno elástico. Com isso, objetivo deste trabalho é indicar a melhor relação entre tempo, temperatura, retorno elástico e propriedades mecânicas para a liga de alumínio AA7475 submetida a um carregamento de flexão em condições de conformação sob fluência e envelhecimento.

\section{MATERIAIS E MÉTODOS}

O material utilizado neste trabalho é a liga de alumínio AA7475 tratável termicamente e que, portanto, atende aos requisitos necessários para à aplicação do processo CAF. Foram produzidos 10 corpos de prova para avaliar a influência dos parâmetros de tratamento no retorno elástico. Esses corpos foram submetidos a um carregamento de flexão em condições de conformação sob fluência e envelhecimento (CAF). Nesse processo, a liga de alumínio utilizada foi previamente solubilizada, temperada e posteriormente carregada elasticamente por flexão, conforme Figura 2 abaixo, seguido de um aquecimento nas temperaturas e tempos de tratamento térmico de envelhecimento pré-estabelecidos: $120^{\circ} \mathrm{C} / 12 \mathrm{horas}$, $150^{\circ} \mathrm{C} / 12$ horas, $120^{\circ} \mathrm{C} / 24$ horas, $150^{\circ} \mathrm{C} / 24$ horas, $120^{\circ} \mathrm{C} / 48$ horas e $150^{\circ} \mathrm{C} / 48$ horas. 


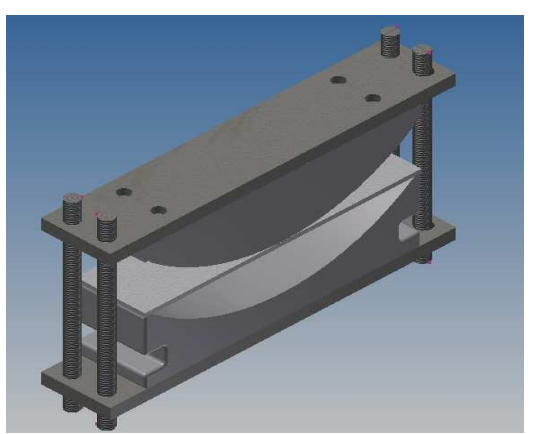

Figura 2: Dispositivo de flexão

Após os ensaios de flexão à quente serem conduzidos, os corpos de prova obtiveram uma flecha final correspondente ao carregamento imposto e as etapas desse processo de conformação que foram realizadas com o dispositivo projetado (para este trabalho) são mostradas através da Figura 3. Para a aquisição de dados que permitam avaliar como os corpos de provas deformaram e assim estabelecer um raio de curvatura e flecha final adquiridos pelo material ao término do carregamento, utilizou-se uma tridimensional Mitutoyo QM Measure $353 \mathrm{com}$ precisão de medição de 3,0 $\mu$ m (Figura 4) disponível no Laboratório de Metrologia do Centro Universitário da FEl. Ao finalizar esta etapa, foram medidas as durezas dos corpos de provas utilizando o durômetro Wilson na escala Vickers (Figura 5).

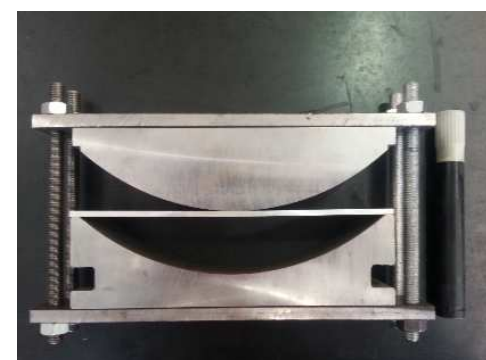

Etapa 1

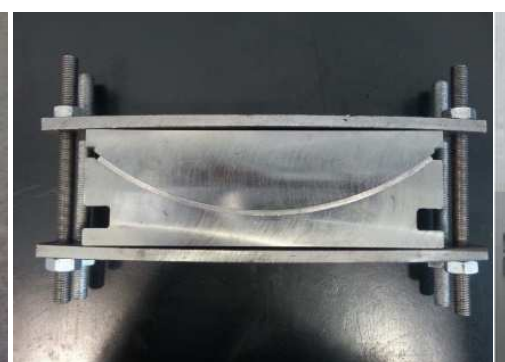

Etapa 2

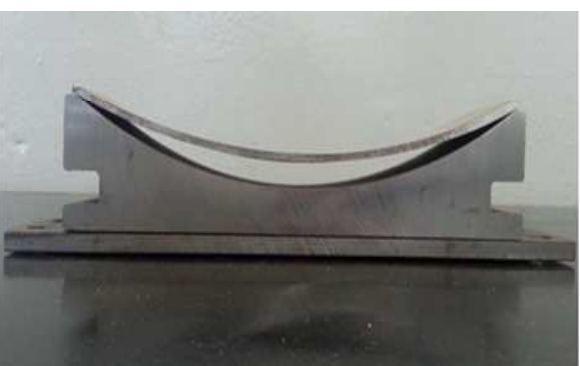

Etapa 3

Figura 3: Etapas do processo de conformação sob fluência.
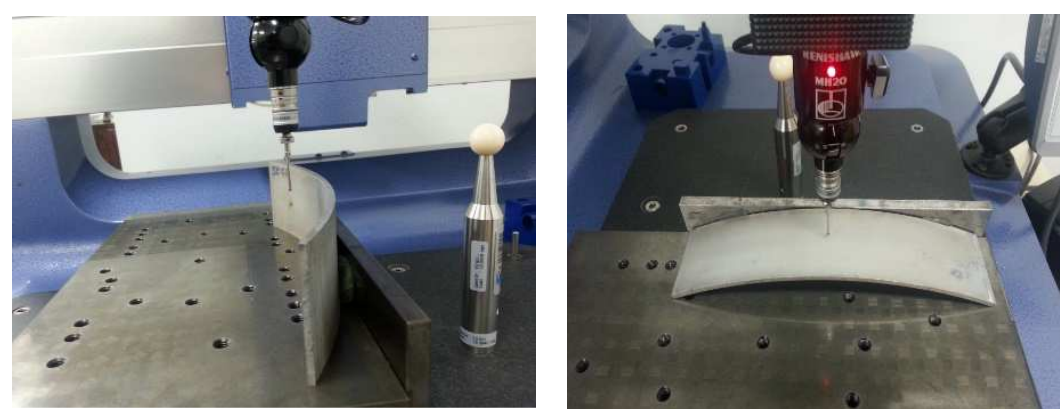

Figura 4: Disposição da peça para obtenção do raio de curvatura e flecha final obtida.
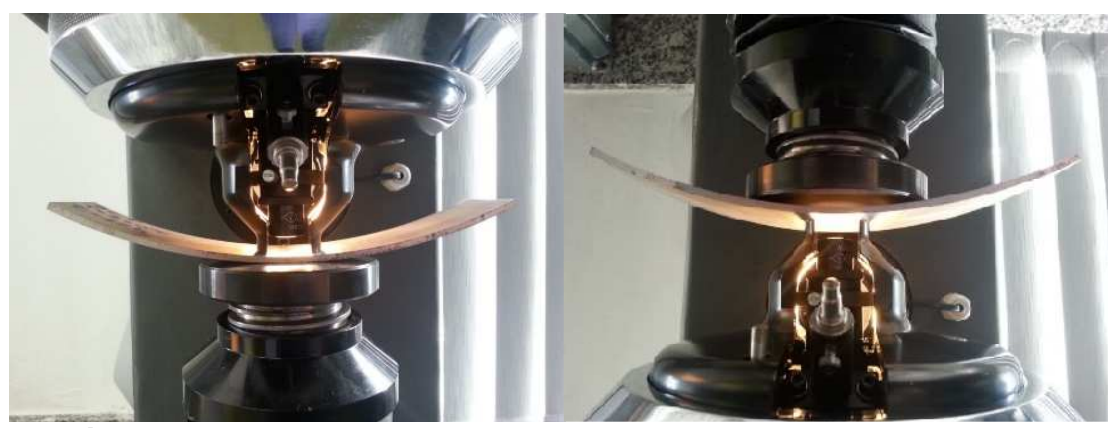

Figura 5: Disposição da peça para obtenção da dureza Vickers. 


\section{RESULTADOS E DISCUSSÃO}

Na secão 1.0 deste trabalho mostrou-se através da Figura 1 a definição do retorno elástico, o qual é quantificado por um fator $R$, em que tem-se a relação de $\delta_{\text {Máx }}$ (distância formada entre a peça que foi ensaiada e a superfície da ferramenta de conformação) com $\delta o$ (distância da peça de trabalho até a ferramenta de conformação), de acordo com a equação 1 abaixo.

\section{R=Retorno Elástico $(\%)=100\left(\delta_{M a ́ x} / \delta o\right)$}

Para um melhor entendimento quanto ao método de cálculo estabelecido, serão exemplificados como foi obtido o retorno elástico (Equação 2 e 3), à partir da média das flechas finais obtidas com a tabela 1 , para $07^{\circ}$ e $8^{\circ}$ corpo de prova que foram submetidos a uma temperatura de envelhecimento de $120^{\circ} \mathrm{C}$ e $150^{\circ} \mathrm{C}$ respectivamente, em um tempo pré-estabelecido de 12 horas.

Como pode ser visto na tabela 1, ao fixar o tempo de envelhecimento em 12 horas e variando a temperatura de $120^{\circ} \mathrm{C}$ para $150^{\circ} \mathrm{C}$ foi obtido para $07^{\circ}$ corpo de prova uma média do diâmetro e flecha final de $426 \mathrm{~mm}$ e $25,55 \mathrm{~mm}$, respectivamente. Para o $8^{\circ}$ corpo de prova analisado, foi obtido uma média do diâmetro de $419 \mathrm{~mm}$ e para flecha final uma média de $26 \mathrm{~mm}$. Com isso é possível visualizar, a ocorrência de diminuição do retorno elástico ao aumentar a temperatura de envelhecimento, reafirmando que quanto menor tempo ou temperatura maior o retorno elástico da peça (ou seja, quanto maior for o retorno elástico da peça mais próxima essa estará do seu formato original antes da conformação).

Ret. Elást. $(\%) 7^{\circ} \mathrm{cp}=100\left(\frac{32,5-25,55}{32,5}\right)=21,4 \%$

Ret. Elást. $(\%) 8^{\circ} \mathrm{cp}=100\left(\frac{32,5-26}{32,5}\right)=20,0 \%$

Tabela 1: Perfil de curvatura e flechas dos corpos de prova ensaiados a $120^{\circ}$ (12horas) e $150^{\circ}$ (12horas).

\begin{tabular}{|c|c|c|c|c|c|c|}
\hline \multicolumn{7}{|c|}{ Perfil de curvatura } \\
\hline \multicolumn{7}{|c|}{ Tempo de envelhecimento: $12 \mathrm{~h}$} \\
\hline \multirow{2}{*}{ CP } & \multirow{2}{*}{$\begin{array}{l}\text { Temperatura de } \\
\text { envelhecimento }\end{array}$} & \multicolumn{3}{|c|}{ Diâmetro [mm] } & \multirow{2}{*}{ Média [mm] } & \multirow{2}{*}{$\begin{array}{c}\text { Desvio } \\
\text { Padrão [\%] }\end{array}$} \\
\hline & & 1 & 2 & 3 & & \\
\hline 7 & $120^{\circ} \mathrm{C}$ & 425,7 & 426,2 & 426 & 426 & 0,25 \\
\hline 8 & $150^{\circ} \mathrm{C}$ & 419 & 420 & 418 & 419 & 1 \\
\hline \multicolumn{7}{|c|}{ Análise da Flecha } \\
\hline \multirow{2}{*}{ CP } & \multirow{2}{*}{$\begin{array}{l}\text { Temperatura de } \\
\text { envelhecimento }\end{array}$} & \multicolumn{3}{|c|}{ Flecha [mm] } & \multirow{2}{*}{ Média [mm] } & \multirow{2}{*}{$\begin{array}{c}\text { Desvio } \\
\text { Padrão [\%] }\end{array}$} \\
\hline & & 1 & 2 & 3 & & \\
\hline 7 & $120^{\circ} \mathrm{C}$ & 25,6 & 25,55 & 25,54 & 25,55 & 0,03 \\
\hline 8 & $150^{\circ} \mathrm{C}$ & 26 & 26 & 26 & 26 & 0 \\
\hline
\end{tabular}

A tabela 2 a seguir, auxiliou na construção do gráfico representado com a Figura 6, referente aos parâmetros de tratamento no retorno elástico da liga de alumínio estudada. 
Tabela 2: Dados para aquisição do gráfico de variação do retorno elástico (springback) em função do tempo.

\begin{tabular}{|c|c|c|c|c|}
\hline Tempo (h) & Ret. Elást. (\%) $/ \mathbf{1 2 0}^{\circ} \mathbf{C}$ & Ret. Elást. (\%) $/ \mathbf{1 5 0}^{\circ} \mathbf{C}$ & [HV] $\mathbf{1 2}{ }^{\circ} \mathbf{C}$ & [HV] $150^{\circ} \mathbf{C}$ \\
\hline 12 & 21,4 & 20,00 & 193 & 195 \\
\hline 24 & 19,69 & 15,51 & 196 & 198 \\
48 & 17,00 & 13,8 & 207 & 191 \\
\hline
\end{tabular}

Após a realização dos cálculos envolvendo os pares de temperaturas e tempos de tratamento térmico de envelhecimento pré-estabelecidos: $120^{\circ} \mathrm{C} / 12 \mathrm{horas}$, $150^{\circ} \mathrm{C} / 12$ horas, $120^{\circ} \mathrm{C} / 24$ horas, $150^{\circ} \mathrm{C} / 24$ horas, $120^{\circ} \mathrm{C} / 48$ horas e $150^{\circ} \mathrm{C} / 48$ horas, foi possível avaliar de forma mais detalhada, por meio da Figura 6 abaixo, os efeitos dos parâmetros mencionados no retorno elástico e indicar a melhor relação entre tempo, temperatura, retorno elástico e propriedades mecânicas para a liga de alumínio AA7475 submetida a um carregamento de flexão em condições de conformação sob fluência e envelhecimento. $O$ gráfico refere-se a variação do retorno elástico (springback) em função do tempo para a liga de alumínio AA7475 submetida a um carregamento de flexão durante o envelhecimento realizado em duas temperaturas diferentes $\left(120^{\circ} \mathrm{C}\right.$ e $\left.150^{\circ} \mathrm{C}\right)$. Portanto é possível concluir que estas análises comparativas condizem com a revisão bibliográfica, pois foi possível observar que com a diminuição do tempo ou temperatura de conformação há um aumento do retorno elástico. Por fim, quanto maior o retorno elástico da peça, mais próxima ela estará do seu formato original antes da conformação.

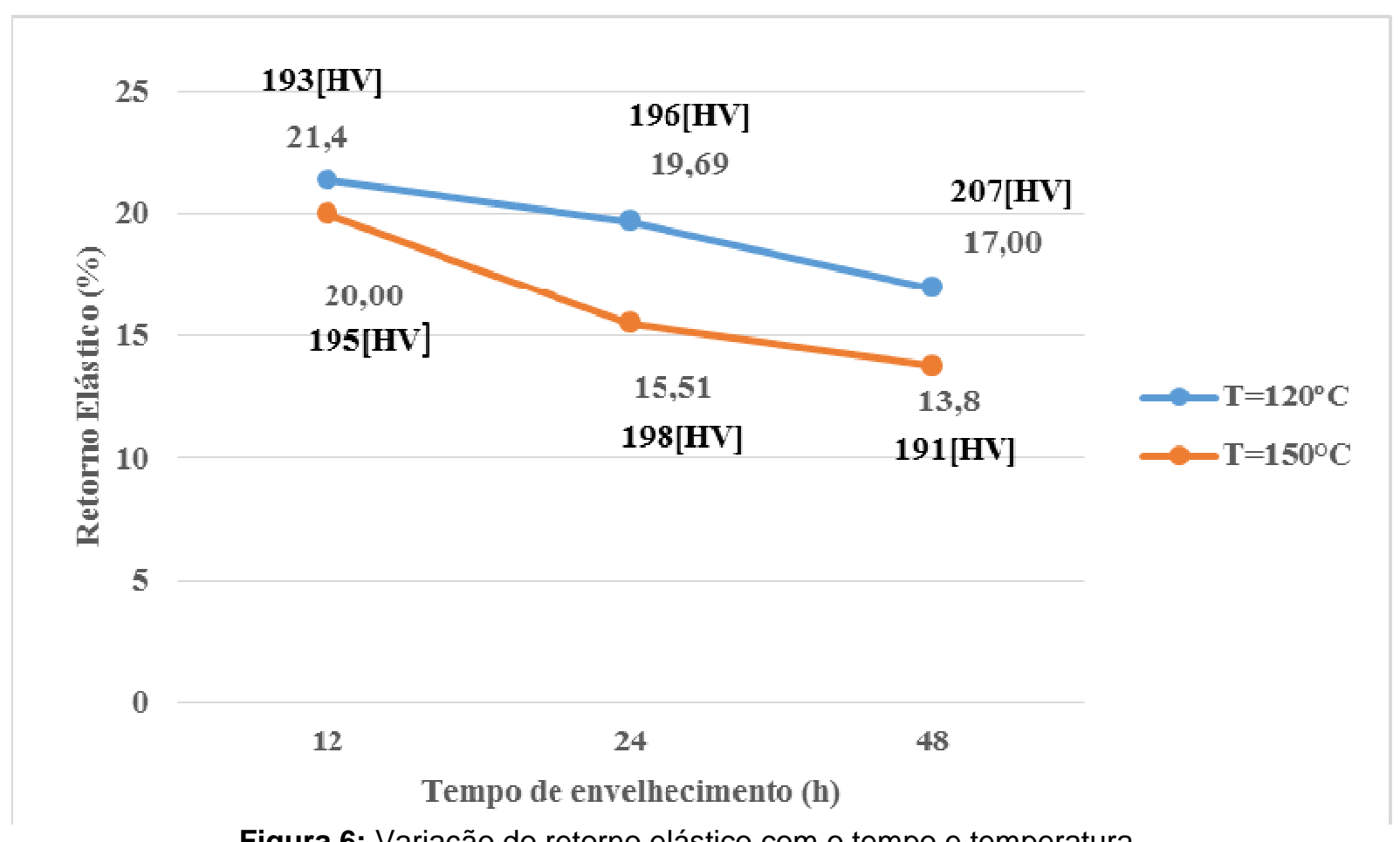

Figura 6: Variação do retorno elástico com o tempo e temperatura.

\section{CONCLUSÃo}

Por meio dos ensaios realizados na liga de alumínio AA7475 submetida a um carregamento de flexão em condições de conformação sob fluência e envelhecimento, analisaram-se os seguintes parâmetros de tratamento no retorno 
elástico: Tempo, temperatura, retorno elástico e propriedades mecânicas. Os dados obtidos aproximam-se das literaturas consultadas para a elaboração deste trabalho. Ao iniciar o processo de envelhecimento, a partir da liga solubilizada, ocorre o fenômeno de precipitação e, consequentemente um endurecimento. Em baixas temperaturas, o envelhecimento ocorre lentamente, necessitando, portanto, de um tempo excessivo para atingir aumentos de dureza e resistência mecânica. Entretanto, em temperaturas elevadas, o processo de envelhecimento é acelerado, porém atingem níveis de dureza e resistência mecânica inferiores quando submetido a baixas temperaturas. Esta afirmação pode ser comprovada ao se comparar os resultados da Figura 6 para o tempo de 48 horas. Para a temperatura de $120^{\circ} \mathrm{C}$ a dureza obtida foi de $207 \mathrm{HV}$ e o retorno elástico de $17 \%$. Para a temperatura de $150^{\circ} \mathrm{C}$ a dureza obtida foi de $191 \mathrm{HV}$ e o retorno elástico de $13,8 \%$. Nota-se portanto, que o retorno elástico é inversamente proporcional ao par, tempo e temperatura.

Em relação à dureza, é possível observar que quando o material é submetido a uma temperatura de $120^{\circ} \mathrm{C}$ obtém-se um aumento significativo de dureza Vickers com o passar do tempo (atribuída a uma precipitação refinada) e a $150^{\circ} \mathrm{C}$ não ocorre esse aumento significativo dessa propriedade mecânica (atribuído a uma precipitação mais grosseira). Aqui vale a mesma observação da Figura 6 . Ao se comparar as durezas para a temperatura de $150^{\circ} \mathrm{C}$ nos tempos de $24 \mathrm{~h}$ e $48 \mathrm{~h}$, percebe-se uma queda de dureza, indicando que ocorreu um superenvelhecimento da liga.

Finalmente, após os ensaios de flexão foi possível obter boa qualidade dimensional final dos corpos de prova por meio da experimentação do processo CAF.

\section{Agradecimentos}

Ao Centro Universitário da FEI pelo patrocínio do projeto e concessão de bolsa de iniciação científica e aos técnicos do Laboratório de Materiais (LabMat-FEl).

\section{REFERÊNCIAS}

1 Jeshvaghani, R.A., Emami, M., Shahverdi, H.R., Hadavi, S.M.M. Effects of time and temperature on creep forming. Materials Sciences and Engineering A 528 (2011), 87958799.

2 Zhan, L., Lin, J., Dean, T.A., A review of the development of creep age forming Experimentation, modelling and applications, International Journal of Machine Tools \& Manufacture 51 (2011).

3 Holman, M.C., Autoclave age forming large aluminum aircraft panels, Journal of Mechanical Working Technology 20 (1989), 477-488 p.

4 Jeunechamps, P.P., Ho, K.C., Lin, J., Ponthot, J.P. \& Dean, T. A. - A closed form technique to predict springback in creep age-forming, Int. Journal of Mechanical Sciences 48, 621-9, 2006.

5 Marcello Vertamatti Mergulhão. Determinação Experimental de curvas de envelhecimento e de fluência para a liga de alumínio AA7050 sob flexão [projeto de iniciação científica (PBIC082/12)], São Bernardo do Campo: Centro Universitário da $\mathrm{FEl} ; 2012 / 2013$. 